\title{
Erratum to: Psychological distress, quality of life, symptoms and unmet needs of colorectal cancer survivors near the end of treatment
}

\author{
Lahiru Russell $^{1}$ - Karla Gough ${ }^{1}$ - Allison Drosdowsky ${ }^{1}$ - Penelope Schofield ${ }^{1,2,3}$. \\ Sanchia Aranda ${ }^{1,2,3} \cdot$ Phyllis N. Butow $^{4,5}$ • Jennifer A. Westwood ${ }^{1}$ • \\ Mei Krishnasamy ${ }^{1,2,3}$ • Jane M. Young ${ }^{4,6}$ • Jo Phipps-Nelson ${ }^{1}$ • Dorothy King ${ }^{1}$ • \\ Michael Jefford ${ }^{1,2,3,7}$
}

Published online: 14 May 2015

(C) Springer Science+Business Media New York 2015

\section{Erratum to: J Cancer Surviv \\ DOI 10.1007/s11764-014-0422-y}

The authors would like to correct the following errors in the original publication:

1. In the first line below the heading "Other data sources", the correct text should read as "Prof. King, University of Sydney" (earlier published as "The lead investigator, University of Sydney").

2. In the eighth line below the same heading, the correct text should read as "No attempt was made to match the general population sample to the SurvivorCare sample on factors predictive of poorer outcomes (age, sex and marital status) [26]" (earlier published as "As sample characteristics from the general population were unavailable, it was not possible to match them with the SurvivorCare sample on factors predictive of poorer outcomes (age, sex and marital status) [26]").

The online version of the original article can be found at http://dx.doi.org/ $10.1007 /$ s11764-014-0422-y.

Michael Jefford

michael.jefford@petermac.org

1 Department of Cancer Experiences Research, Peter MacCallum Cancer Centre, East Melbourne, Melbourne, VIC 3002, Australia

2 Sir Peter MacCallum Department of Oncology, The University of Melbourne, Parkville, Melbourne, VIC 3010, Australia

3 Faculty of Medicine, Dentistry and Health Services, The University of Melbourne, Parkville, Melbourne, VIC 3010, Australia
$4 \quad$ Surgical Outcomes Research Centre, University of Sydney and Sydney South West Area Health Service, Missenden Rd, PO Box M157, Sydney, NSW 2050, Australia

5 Centre for Medical Psychology and Evidence-based Decision Making (CeMPED), School of Psychology, University of Sydney, A18, Sydney, NSW 2006, Australia

6 School of Public Health, University of Sydney, A27, Sydney, NSW 2006, Australia

Peter MacCallum Cancer Centre, Locked Bag 1, A’Beckett Street, Melbourne, VIC 8006, Australia 